\title{
Crossing barriers: a multidisciplinary approach to children and adults with young-onset movement disorders
}

\author{
Martje E. van Egmond ${ }^{1,2^{*}+}$ (D) Hendriekje Eggink ${ }^{1+}$, Anouk Kuiper ${ }^{1}$, Deborah A. Sival ${ }^{3}$, \\ Corien C. Verschuuren-Bemelmans ${ }^{4}$, Marina A. J. Tijssen ${ }^{1}$ and Tom J. de Koning ${ }^{1,3,4}$
}

\begin{abstract}
Background: Diagnosis of less common young-onset movement disorders is often challenging, requiring a broad spectrum of skills of clinicians regarding phenotyping, normal and abnormal development and the wide range of possible acquired and genetic etiologies. This complexity often leads to considerable diagnostic delays, paralleled by uncertainty for patients and their families. Therefore, we hypothesized that these patients might benefit from a multidisciplinary approach. We report on the first 100 young-onset movement disorders patients who visited our multidisciplinary outpatient clinic.
\end{abstract}

Methods: Clinical data were obtained from the medical records of patients with disease-onset before age 18 years. We investigated whether the multidisciplinary team, consisting of a movement disorder specialist, pediatric neurologist, pediatrician for inborn errors of metabolism and clinical geneticist, revised the movement disorder classification, etiological diagnosis, and/or treatment.

Results: The 100 referred patients (56 males) had a mean age of $12.5 \pm 6.3$ years and mean disease duration of $9.2 \pm 6$. 3 years. Movement disorder classification was revised in 58/100 patients. Particularly dystonia and myoclonus were recognized frequently and supported by neurophysiological testing in 24/29 patients. Etiological diagnoses were made in 24/71 (34\%) formerly undiagnosed patients, predominantly in the genetic domain. Treatment strategy was adjusted in 60 patients, of whom 43 (72\%) reported a subjective positive effect.

Conclusions: This exploratory study demonstrates that a dedicated tertiary multidisciplinary approach to complex young-onset movement disorders may facilitate phenotyping and improve recognition of rare disorders, with a high diagnostic yield and minimal diagnostic delay. Future studies are needed to investigate the cost-benefit ratio of a multidisciplinary approach in comparison to regular subspecialty care.

Keywords: Multidisciplinary, Young-onset movement disorders, Diagnosis, Dystonia, Myoclonus, Clinical phenotyping

\section{Background}

Young-onset movement disorders (YMDs) is a relatively new field in neurology, comprising clinical neurological syndromes presenting with involuntary movements manifesting before the age of 18 . As with movement disorders (MDs) in adults, YMDs are subdivided into hyperkinetic

\footnotetext{
* Correspondence: m.e.van.egmond@umcg.nl

${ }^{\dagger}$ Equal contributors

'Department of Neurology, University of Groningen, University Medical Center Groningen, Groningen, the Netherlands

${ }^{2}$ Department of Neurology, Ommelander Ziekenhuis Groningen, Delfzijl and Winschoten, PO Box 30.001, 9700, RB, Groningen, the Netherlands Full list of author information is available at the end of the article
}

movements (dystonia, myoclonus, chorea, ballism, tremor and tics), hypokinetic (parkinsonism) movements, and ataxia [1-5]. Recognition of common YMDs, such as tics and stereotypies, is usually straightforward for most clinicians. However, diagnosis of less common and more complex YMDs, such as disorders presenting primarily with myoclonus or dystonia, is often difficult, both for pediatric and adult neurologists $[1,6,7]$.

The recognition and classification of YMDs present some unique challenges. Firstly, YMDs are often embedded in a complex clinical phenotype. For example, the occurrence of mixed MDs (more than one MD present) 
or co-existence of a variety of symptoms such as psychomotor retardation or behavioral abnormalities are commonly seen $[5,8,9]$. Secondly, in young children the developing nervous system may produce a variety of motor patterns that would be labeled as pathologic in older children and adults, but are simply a manifestation of brain immaturity in younger patients [1]. For instance, chorea is a normal feature in healthy infants and toddlers, and (subtle) signs of overflow dystonia and ataxia are found in healthy children up till the age of 12 years or even older [10, 11]. Finally, YMDs can be caused by a broad spectrum of both acquired and genetic disorders, including infections, auto-antibody and auto-immune disorders, as well as rare metabolic disorders and other inherited defects [7, 12-14].

The complexity of the diagnosis and management of YMDs is becoming increasing clear, which has resulted in a growing number of specialized pediatric neurologists. Despite this development, the diagnostic process in complex YMDs often remains challenging, a burden for patients and their families, and costly for our health care system as patients often remain undiagnosed for many years $[1,6,7,14,15]$. This has been reflected in a recent study in a tertiary referral center that showed a mean delay of diagnosis of $11.1 \pm 12,5$ years in a cohort of 260 patients with non-tic YMDs [7].

In other heterogeneous or rare diseases in children such as epilepsy or neuromuscular disorders, a beneficial effect of a multidisciplinary approach has been reported. [16-20] We hypothesized that such an approach might be a possibility to tackle the complexity of children and young adults with MDs. A multidisciplinary team may enable to overcome the three difficulties experienced in this patient group: a complex clinical phenotype (movement disorder specialist), the variety of motor patterns produced by the developing brain (pediatric neurologist), and a broad spectrum of both acquired and genetic disorders (pediatrician for inborn errors of metabolism and a clinical geneticist).

In this exploratory study, we report on the first 100 patients with YMDs who visited our multidisciplinary outpatient clinic. Our aim was to share our experience of a new multidisciplinary approach in terms of changes in MD classification, diagnostic yield and targeted treatment strategies.

\section{Methods}

\section{Design and setting of the study}

In this retrospective, single center, observational study we evaluated the first 100 patients who visited our multidisciplinary outpatient clinic for YMDs. It was situated in a tertiary referral center, the University Medical Center Groningen, in the Netherlands. The study was performed according to the ethical standards and regulations of our institute.

\section{Patients}

All patients had a confirmed or suspected MD with an onset before the age of 18 years and were referred for an expert opinion regarding MD classification, etiology or treatment of involuntary movements (Table 1).

\section{Multidisciplinary outpatient clinic}

The clinic was initiated in 2012 with a team consisting of an adult neurologist specialized in MDs (MT), a pediatric neurologist specialized in developmental neurology and YMDs especially ataxia (DS), a pediatrician specialized in metabolic diseases (TK) and a clinical neuro-geneticist $(\mathrm{CV})$. In addition, clinical fellows in movement disorders and residents attend the clinic to gain skills and knowledge from the different medical specialties involved as part of their clinical training.

The pediatrician for inborn errors of metabolism received the referrals as the coordinating medical specialist, which were subsequently discussed within the team. Prior to the consultation, referral letters and medical reports containing previous diagnostic and treatment strategies were read carefully by the clinical fellow, who sent a summary to all team members.

During the consultation, patients were seen by all team members at once. In a separate meeting, the team members reviewed the video images, discussed the movement disorder classification and the results of the additional investigations, and developed joint diagnostic and therapeutic recommendations. In all cases the team members reached consensus. The main diagnostic steps were laboratory investigations, (neuro-)imaging, clinical neurophysiology or genetic testing. The key therapeutic options comprised pharmacological treatment, botulinum toxin injections, paramedical interventions (e.g. physiotherapy, occupational therapy, speech therapy), ketogenic diet, and deep brain stimulation.

The primary purpose of the multidisciplinary team was not to take over the clinical care provided by the referring medical specialist, but preferably to see a patient once and provide an all-in-one expert opinion. The presence of the clinical geneticist enabled direct genetic counseling in case genetic testing was considered. Results of additional investigations and genetic diagnostics were shared with the patient or caregivers by one of the team members via a follow-up consult or, if preferred by the family, by a telephone consultation. The team aimed to leave further management and follow-up to the referring specialist, but in case of unresolved issues patients were welcome to return to the multidisciplinary outpatient clinic. 
Table 1 Baseline characteristics

\begin{tabular}{|c|c|}
\hline \multicolumn{2}{|l|}{ Patient characteristics } \\
\hline Sex (male/female) & $56 / 44$ \\
\hline Age (years) ${ }^{a}$ & $12.5 \pm 6.3 ; 1-33$ \\
\hline Age at symptom-onset (years) ${ }^{a}$ & $3.3 \pm 4.6 ; 0-18$ \\
\hline Duration of symptoms (years) ${ }^{a}$ & $9.2 \pm 6.3 ; 1-32$ \\
\hline \multicolumn{2}{|l|}{ Referral questions } \\
\hline Movement disorder classification & 50 \\
\hline Etiology & 38 \\
\hline Treatment & 42 \\
\hline \multicolumn{2}{|l|}{ MD classification } \\
\hline Ataxia & 9 \\
\hline Dystonia & 32 \\
\hline Myoclonus & 11 \\
\hline Other ${ }^{\mathrm{b}}$ & 12 \\
\hline Unclassified & 36 \\
\hline \multicolumn{2}{|l|}{ Etiological diagnosis } \\
\hline Inherited etiologies & 17 \\
\hline \multicolumn{2}{|l|}{ Monogenic } \\
\hline ARX mutation & 1 \\
\hline Ataxia telangiectasia & 1 \\
\hline Coffin Lowry syndrome & 1 \\
\hline Glutaric aciduria type 1 & 2 \\
\hline GLI2 mutation & 1 \\
\hline GOSR2 mutation & 1 \\
\hline GTPCH deficiency (DYT5) & 1 \\
\hline Proprionacidemia & 1 \\
\hline SCN1A mutation & 2 \\
\hline THAP1 mutation (DYT6) & 2 \\
\hline TITF1 mutation & 1 \\
\hline \multicolumn{2}{|l|}{ Structural chromosomal abnormality } \\
\hline $\begin{array}{l}\text { Microdeletion 19p13.2p13.13 } \\
\text { (NFIX and CACNA1A gene) }\end{array}$ & 1 \\
\hline Partial deletion chromosome 7q (SCGE gene) & 1 \\
\hline Uniparental disomia chromosome 7 (SCGE gene) & 1 \\
\hline Acquired etiologies & 12 \\
\hline Infectious & 2 \\
\hline Perinatal asphyxia & 9 \\
\hline Functional & 2 \\
\hline
\end{tabular}

${ }^{\mathrm{a}}$ Age in years \pm standard deviation; range

${ }^{b}$ Chorea, tics, tremor, parkinsonism and if no MD was present Abbreviations: ARX, Aristaless related homeobox; GOSR2, Golgi SNAP receptor complex member 2; GTPCH, Guanosine Triphosphate Cyclohydrolase; SCN1A, sodium channel voltage gated type I alpha subunit; TITF1, Thyroid transcription factor-1; NFIX, nuclear factor I/X; CACNA1A, calcium channel voltage-dependent, $\mathrm{P} / \mathrm{Q}$ type, alpha $1 \mathrm{~A}$ subunit; $S C G E$, epsilon-sarcoglycan

\section{Data collection}

We evaluated the first 100 patients who visited our multidisciplinary clinic for YMDs between June 2012 and May 2014. Medical records were reviewed for patient characteristics and previous phenotypical classifications. The severity of the YMDs present was assessed by the team members using the global clinical impression scale of severity (GCI-S). This commonly used 7-point scale enables a clinician to rate the extent movement disorders with no movement disorder (1), slight (2), mild (3), moderate (4), marked (5), severe (6), and among the most severest (7) [21]. We compared the classification of the most prominent MD and etiological diagnosis before and after assessment by the multidisciplinary team. In addition, we studied the treatment strategies and whether the patients or their caregivers reported any positive effects of therapies 3-6 months after initiation. Since many patients were not under our primary care, and/or living at a distance from our center, we performed follow-up using a semi-structured interview during a telephone consultation. Patients and/or caregivers were asked (1) whether they experienced benefit with regard to motor symptoms, (2) since when they experienced this, (3) extent of improvement (none/slight/moderate/good), and (4) if any adverse effects were present.

\section{Results}

\section{Patient characteristics}

A total of 56 male and 44 female patients visited the multidisciplinary clinic (Table 1). Patients had a mean age of 12.5 years (SD 6.3) and a mean duration of symptoms of 9.2 years (SD 6.3). Referring specialists were predominantly pediatric neurologists, pediatricians and rehabilitation doctors with questions concerning the MD classification, etiology or treatment options. We had 36 patients referred with an unclassified MD, documented as dyskinesias, trembling, involuntary movements, or restlessness. A confirmed etiological diagnosis (17 inherited, 12 acquired) already explained the phenotype of 29 patients upon referral.

\section{Movement disorder classification}

Mean severity of the MDs present was $4.3 \pm 1.7$ on the global clinical impression scale (range 1-7), corresponding with a moderate to marked MD severity. The multidisciplinary team revised the initial classification in 58/ 100 patients (Table 2). These revisions reduced the number of patients with an unclassified MD from 36 down to 4. Compared to the referring clinicians, the team more frequently classified the patients' involuntary movements as dystonia (from 32 to 41 ) and myoclonus (from 11 to 31). The number of ataxic and tremor patients dropped (from 9 to 1 and 6 to 1 , respectively), 
Table 2 Overview of classification of most prominent MD before and after visiting the multidisciplinary outpatient clinic

\begin{tabular}{|c|c|c|c|c|c|c|c|}
\hline & & \multicolumn{6}{|c|}{ Observed MD classification by the multidisciplinary team } \\
\hline & & Dystonia & Myoclonus $^{a}$ & Ataxia & Other $^{\mathrm{b}}$ & Unclassified & Total \\
\hline \multirow[t]{6}{*}{ Referral MD classification } & Dystonia & 26 & 1 & 0 & 4 & 1 & 32 \\
\hline & Myoclonus $^{\mathrm{a}}$ & 0 & 10 & 0 & 1 & 0 & 11 \\
\hline & Ataxia & 0 & 8 & 0 & 1 & 0 & 9 \\
\hline & Other ${ }^{b}$ & 2 & 5 & 0 & 5 & 0 & 12 \\
\hline & Unclassified & 13 & 7 & 1 & 12 & 3 & 36 \\
\hline & Total & 41 & 31 & 1 & 23 & 4 & 100 \\
\hline
\end{tabular}

Isolated myoclonus, myoclonus ataxia and myoclonus dystonia

${ }^{\mathrm{b}}$ Comprises chorea, tics, tremor, parkinsonism and if no MD was present

whereas the number of patients with chorea increased (from 4 to 6). The multidisciplinary team observed no MDs in eleven patients (e.g. the movements were related to agitation or caused by behavioral abnormalities). Simultaneous non-invasive surface electroencephalography/ electromyography (EEG/EMG) was performed in 29 predominantly myoclonic patients and this confirmed or supported the MD classification observed by the team in 24/29 patients. In the remaining five cases, EEG/EMG was not conclusive due to an absence of symptoms during registration $(n=3)$ or the patient being unable to comply with the registration protocol $(n=2)$.

\section{Associated neurological and non-neurological features}

Only 26/100 patients presented with a (mixed) MD without associated features, whereas the majority of patients also had additional neurological symptoms $(n=35)$, non-neurological symptoms $(n=9)$ or both $(n=30)$. The most important additional features were intellectual disability, epilepsy, spasticity, skin abnormalities, deafness, dysmorphias, and skeletal and growth abnormalities.

\section{Etiological diagnosis}

At presentation, 29/100 patients had a confirmed genetic or acquired cause explaining their phenotype (Table 1). The multidisciplinary team established a diagnosis in 24 additional patients (Table 3), particularly in the genetic domain, where the number of diagnoses more than doubled from 17 to 37. Monogenetic etiologies were found using single-gene testing in nine cases, by targeted resequencing in three cases and using whole exome sequencing in five cases. Biochemical testing led to a diagnosis of non-ketotic hyperglycinemia in one case in which confirmation of the molecular defect is still pending.

Among the acquired causes, oral contraceptiveinduced chorea was diagnosed in one patient and three patients turned out to have functional MDs. Despite an increase in confirmed etiological diagnoses from 29 to 53, we still had 35 patients categorized with a suspected genetic diagnosis (defined as strong suspicion of a genetic cause based on a severe clinical phenotype, early onset, family history, and absence of any of the known acquired causes). In these cases, multiple genetic tests, including whole exome sequencing, have not yet

Table 3 Confirmed etiological diagnoses after assessment by the multidisciplinary team

\begin{tabular}{ll}
\hline Diagnosis & $\mathbf{N}$ \\
\hline Inherited etiologies & 20 \\
Monogenic & 1 \\
ACTB mutation & 1 \\
CTNNB1 mutation & 1 \\
GLRA1 mutation & 6 \\
GOSR2 mutation & 1 \\
HSD17B10 mutation & 1 \\
MECP2 mutation & 1 \\
OFD-1 mutation & 1 \\
OTC-deficiency & 1 \\
PRRT2 mutation & 1 \\
SPTBN2 mutation & 1 \\
TH mutation & 1 \\
TITF-1 mutation & 1 \\
Laboratory abnormalities & 1 \\
Non-ketotic hyperglycinemia & 1 \\
Syndrome diagnosis & 1 \\
Gilles de la Tourette & 1 \\
Linear naevus syndrome & 1 \\
Acquired etiologies & 1 \\
Drug-induced & 1 \\
\hline Functional & 1 \\
\hline
\end{tabular}

Abbreviations: $A C T B$, beta-actin; CTNNB1, catenin (cadherin-associated protein) beta 1; GLRA1, glycine receptor alpha 1; GOSR2, Golgi SNAP receptor complex member 2; HSD17B10, 17beta-hydroxysteroid dehydrogenase type 10; MECP2, methyl CpG binding protein 2; OFD-1, oral-facial-digital syndrome 1; OTC, ornithine carbamoyltransferase; PRRT2, proline-rich transmembrane protein 2; SPTBN2, spectrin beta non-erythrocytic 2; TH, tyrosine hydroxylase; TITF1, thyroid transcription factor-1; HSD17B10 or 2-methyl-3-hydroxybytyryl-CoA dehydrogenase deficiency. 
revealed a causative molecular defect. For 21 of these 35 patients we are awaiting elucidation of the causal mutation in a research setting, the other 14 patients (or their caregivers) decided not to participate in this research.

\section{Treatment strategies}

More than half of the 100 patients (61\%) had not been given any specific treatment for their MD before visiting our clinic. The multidisciplinary team initiated or changed the treatment strategy in $60 / 100$ of the patients. Table 4 gives an overview of changes in the treatment strategy, categorized by MD type. In 30/60 cases (50\%), the new treatment strategy was based on the revised MD classification. In the other 30 patients the team initiated or adjusted the treatment strategy, despite an unchanged MD classification: for example symptomatic treatment with trihexyphenidyl in dystonic cerebral

Table 4 Overview of treatment strategies that were changed by the multidisciplinary team

\begin{tabular}{|c|c|c|c|c|}
\hline $\begin{array}{l}\text { Movement } \\
\text { disorder }\end{array}$ & $\begin{array}{l}\text { Treatment } \\
\text { category }\end{array}$ & $\begin{array}{l}\text { Treatment } \\
\text { specifics }\end{array}$ & $N$ & $\begin{array}{l}\text { Pos } \\
\text { effe }\end{array}$ \\
\hline \multicolumn{5}{|l|}{ Dystonia } \\
\hline & \multicolumn{4}{|l|}{ Pharmacological } \\
\hline & & Clonazepam & 1 & 1 \\
\hline & & Gabapentin & 3 & 3 \\
\hline & & L-dopa & 2 & 1 \\
\hline & & Trihexyphenidyl & 8 & 3 \\
\hline & & Cessation of drug & 1 & 1 \\
\hline & Botulinum toxin & & 5 & 5 \\
\hline & Deep brain stimulation & & 5 & 4 \\
\hline & Paramedical & & 2 & 2 \\
\hline & Total dystonia & & 27 & 20 \\
\hline \multicolumn{5}{|l|}{ Myoclonus } \\
\hline & Pharmacological & Clonazepam & 10 & 10 \\
\hline & Ketogenic diet & & 4 & 1 \\
\hline & Paramedical & & 4 & 2 \\
\hline & Total myoclonus & & 18 & 12 \\
\hline
\end{tabular}

Other

Pharmacological

\begin{tabular}{|c|c|c|c|c|}
\hline & & L-dopa & 4 & 4 \\
\hline & & Acetozolamide & 1 & 1 \\
\hline & & Cessation of drug & 4 & 2 \\
\hline & Botulinum toxin & & 1 & 1 \\
\hline & Paramedical & & 3 & 2 \\
\hline & Total other & & 13 & 10 \\
\hline Difficult & & & 2 & \\
\hline to classity & Pharmacological & L-dopa & 2 & 1 \\
\hline Total & & & 60 & 43 \\
\hline
\end{tabular}

palsy. We advised six patients to stop their medication, which led to unchanged clinical symptoms in two patients and an improvement of symptoms in three others. An example of the latter was advice to stop taking oral contraceptives, which led to an almost complete disappearance of adolescent-onset chorea. In the group of 60 patients who had new or adjusted treatment, $72 \%$ of them or their caregivers reported a positive effect therapy after 3-6 months. Five patients were advised to stop their medication at the 3-6 months evaluation, because of limited benefit and or potential aggravation of other symptoms and side effects, such as effects on mood, behavior or constipation.

\section{Discussion}

To our knowledge, this is the first study describing the experience with a multidisciplinary team approach towards children and adults with YMDs. Based on the results it is likely that patients with YMDs benefit from a multidisciplinary team strategy with regard to MD classification, diagnostic yield and targeted treatment strategies.

The multifaceted nature of YMDs served as an impulse for setting up our multidisciplinary outpatient clinic, because the complexity of YMDs often leads to a time-consuming and burdensome diagnostic process $[1$, $6,7]$. This issue is reflected by a mean symptom duration of $74 \%$ of our patients' life spans, which is in line with the results of a previous study [7]. In $58 \%$ of the patients, the team revised the MD classification or defined another MD as the most prominent clinical symptom. We think this high percentage of revisions may be due to the combined expertise of a pediatric neurologist, trained to distinguish normal developmental from abnormal movements, and a movement disorder specialist, trained to establish the phenomenology of clinical MD syndromes $[1,8]$. Although we are aware that there is no gold standard for clinical MD classification, additional investigations such as EEG/EMG for myoclonus confirmed the clinical diagnosis in $24 / 29$ of our cases [22]. The presence of non-neurological features in 39\% of our YMD cohort underscores the complexity of the clinical presentations in a significant part of this population, and the combined expertise of a pediatrician and a clinical geneticist to include all symptoms, facilitated the diagnostic process.

The team identified a etiological diagnosis in 24/71 (34\%) previously undiagnosed patients, of which 17 were found to have monogenetic disorders. In contrast, in a study with 260 patients non-tic YMDs patients, who were referred to a neurologist specialized in YMDs between 2004 and 2013, a definitive genetic diagnosis was made in $17 \%$. [7]. We realize that the genetic advances of the past decade are likely to have contributed to the higher yield in our sample, however we hypothesize that 
the team's broad and combined expertise has also been an important contributing factor. Furthermore, the diagnostic yield was obtained in a relatively short period of time, as a multidisciplinary team strategy facilitates immediate decision-making in comparison to the normal serial process involving multiple referrals, therefore minimizing the burden for the patients and their families.

After critical appraisal of phenotype and etiology, therapeutic strategies were considered and tailored to individual patient needs. The team gave specific advice on treatment for $60 \%$ of patients, with $72 \%(n=42)$ of them or their caregivers reporting a subjective positive effect of the suggested treatment on follow-up. The effectiveness of treatment was only assessed through a semistructured questionnaire and it was therefore not possible to draw more detailed conclusions on objective and/or long-term outcome measures of its effectiveness. Nevertheless, the large number of patients in which treatment was initiated at our clinic may reflect a potential under-treatment of YMDs, likely to significantly impact the patient's quality of life. The low number of patients that were already treated for their MD is remarkable, in particular when taking into account that the mean MD severity of these 60 cases was significant ( 5 on a scale of 7). Low treatment rates and potential under-treatment have also been reported in MDs in children with inborn errors of metabolism, [13] despite the fact that it has been shown that symptomatic treatment may significantly improve patients' daily functioning and quality of life $[14,23,24]$.

The results of this exploratory study indicate that YMDs patients might benefit from a multidisciplinary team approach in terms of diagnosis and treatment in comparison to the referring specialists. However, interpretation of the results is limited by the lack of a control group of patients' receiving assessment by a pediatric movement disorder specialist, or in comparison to assessments by an alternative team consisting of two or three specialists. Inclusion of such a control group was not feasible in our center. Nevertheless, we think that this single-institution experience indicates that a dedicated multidisciplinary approach to YMDs disorders may facilitate phenotyping and improve recognition of rare disorders.

Notably, in this study, the age at presentation at our outpatient clinic ranged from 1 to 33 years, which is beyond the standard upper limit of 18 years for pediatric care. Distinguishing early-onset from lateronset MDs is useful for diagnostic purposes [3, 4]. However, we believe that the age of symptom-onset in these patients is a more important inclusion criterion than the age at time of referral, especially because long delays between symptom-onset and diagnosis have been reported. [7] Therefore, we propose to consider patients with YMDs as a spectrum, irrespective of the age of referral, and to allow all complex YMD patients to benefit from the combined expertise of a multidisciplinary team, crossing barriers between pediatric and adult neurology.

\section{Conclusion}

In summary, our results suggest that a multidisciplinary approach might help tackle the complexity of diagnosis and managing complex YMDs. Our experience indicates that this approach may improve recognition of rare disorders, with a good diagnostic yield and a minimal diagnostic delay. Future studies are needed to investigate the cost-benefit ratio of a multidisciplinary approach in comparison to regular subspecialty care, preferably using a prospective study design with standardized clinical assessments to systematically evaluate treatment effects.

\section{Abbreviations}

EEG/EMG: Electroencephalography/electromyography; MD: Movement disorder; YMD: Young-onset movement disorder

\section{Acknowledgements}

The authors thank Jackie Senior (University Medical Center Groningen, Department of Genetics) for editing the manuscript, and Maria Fiorella Contarino (Department of Neurology, Leiden University Medical Centre, and Department of Neurology, Haga Teaching Hospital, The Hague, the Netherlands) for her valuable comments on the manuscript.

\section{Funding}

No funding was received for this study.

\section{Availability of data and materials}

The datasets analysed during the current study are not publicly available because these contain information of the medical records of the individual patients, but are available from the corresponding author on reasonable request.

\section{Authors' contributions}

$\mathrm{HE}$ and MvE attended the outpatient clinic, collected and analyzed the data and wrote the first version of the manuscript ( $1 A-C ; 2 A-C$; and $3 A-B)$. AK attended the outpatient clinic as PhD student (1A-C; 3B). DS, CV, MT and TdK formed the multidisciplinary team for the outpatient clinic for young-onset movement disorders (1 A-C, 3B). All authors were involved in the conception and design of the work and interpretation of the data. All authors participated critically revised the manuscript and approved the final version. All authors agree to be accountable for all aspects of the work in ensuring that questions related to the accuracy or integrity of any part of the work are appropriately investigated and resolved.

\section{Ethics approval and consent to participate}

This study was carried out in accordance with the ethical standards and regulations of the Medical Ethical Committee of the University Medical Center Groningen, the Netherlands. All subjects in this study received care-as-usual and the presented data cannot be traced back to individual patients. Therefore this study is not subject to the Law on Medical Scientific Research involving Human Beings (WMO), and written informed consent from the subjects was not required.

\section{Consent for publication}

Not applicable, as the manuscript does not contain any individual person's data.

\section{Competing interests}

The authors declare that they have no competing interests. Full financial disclosures of all authors: Dr. van Egmond received a travel grant from Medtronic; Prof Tijssen received research grants from Fonds Nuts-Ohra, Stichting Wetenschapsfonds Dystonievereniging, Prinses Beatrix Foundation, 
STW Technology society (NeuroSIPE), and Phelps Stichting voor Spastici. Unrestricted grants were received from Allergan Pharmaceuticals, Actelion, Ipsen Pharmaceuticals. An unrestricted grant was received from Medtronic for a dystonia nurse, and from DystonieNet for a teaching course. Dr. de Koning received a research grant from Metakids Foundation, Ride4Kids Foundation, and Metabolic Power Foundation (non-profit) and a research grant from Actelion (for profit). The other authors have indicated they have no financial relationships to disclose.

\section{Publisher's Note}

Springer Nature remains neutral with regard to jurisdictional claims in published maps and institutional affiliations.

\section{Author details}

'Department of Neurology, University of Groningen, University Medical Center Groningen, Groningen, the Netherlands. ${ }^{2}$ Department of Neurology, Ommelander Ziekenhuis Groningen, Delfzijl and Winschoten, PO Box 30.001, 9700, RB, Groningen, the Netherlands. '3epartment of Pediatrics, University of Groningen, University Medical Center Groningen, Groningen, the Netherlands. ${ }^{4}$ Department of Genetics, University of Groningen, University Medical Center Groningen, Groningen, the Netherlands.

Received: 28 December 2017 Accepted: 22 March 2018

Published online: 06 April 2018

\section{References}

1. Singer HS, Mink JW, Gilbert DL, Jankovic J. Movement Disorders in Childhood. 2nd ed. Philadelphia: Saunders Elsevier; 2016.

2. Sanger TD, Chen D, Fehlings DL, et al. Definition and classification of hyperkinetic movements in childhood. Mov Disord. 2010;25:1538-49.

3. Fahn S. Classification of movement disorders. Mov Disord. 2011;26:947-57.

4. Albanese A, Bhatia K, Bressman SB, Delong MR, Fahn S, Fung V, et al. Phenomenology and classification of dystonia: a consensus update. Mov Disord. 2013:28:863-73.

5. Delgado MR, Albright AL. Movement disorders in children: definitions, classifications and grading systems. J Child Neurol. 2003;15(Suppl):1-8.

6. van Egmond ME, Kuiper A, Eggink H, Sinke RJ, Brouwer OF, Verschuuren Bemelmans CC, et al. Dystonia in children and adolescents: a systematic review and a new diagnostic algorithm. J Neurol Neurosurg Psychiatry. 2015;86:774-81.

7. Bäumer T, Sajin V, Münchau A. Childhood-onset movement disorders: a clinical series of 606 cases. Mov Disord Clin Prac. 2017:4:437-40.

8. Abdo WF, van de Warrenburg BPC, Burn DJ, Quinn NP, Bloem BR. The clinical approach to movement disorders. Nat Rev. Neurol. 2010;6:29-37.

9. Sanger TD, Chen D, Fehlings DL, Hallett M, Lang AE, Mink JW, et al. Definition and classification of hyperkinetic movements in childhood. Mov Disord. 2010;25:1538-49.

10. Brandsma R, Spits AH, Kuiper MJ, Lunsing RJ, Burger H, Kremer HP, et al. Ataxia rating scales are age-dependent in healthy children. Dev Med Child Neurol. 2014;56:556-63.

11. Kuiper MJ, Vrijenhoek L, Brandsma R, Lunsing RJ, Burger $H$, Eggink $H$, et al. The Burke-Fahn-Marsden Dystonia Rating Scale is Age-Dependent in Healthy Children. Mov Disord Clin Pract. 2015;3:580-6.

12. Gascon GG, Ozand PT, Brismar J. Movement disorders in childhood organic acidurias. Clinical, neuroimaging, and biochemical correlations. Brain \& Development. 1994;16(Suppl):94-103.

13. García-Cazorla A, Wolf NI, Serrano M, Pérez-Dueñas B, Pineda M, Campistol J, et al. Inborn errors of metabolism and motor disturbances in children. J Inherit Metab Dis. 2009;32:618-29.

14. Eggink H, Kuiper A, Peall KJ, Contarino MF, Bosch AM, Post B, et al. Rare inborn errors of metabolism with movement disorders: a case study to evaluate the impact upon quality of life and adaptive functioning. Orphanet J Rare Dis. 2014;9:177.

15. Bertram KL, Williams DR. Diagnosis of dystonic syndromes - a new eightquestion approach. Nat Rev. Neurol. 2012;8:275-83.

16. Shapiro BS, Cohen DE, Covelman KW, Howe CJ, Scott SM. Experience of an interdisciplinary pediatric pain service. Pediatrics. 1991:88:1226-32.

17. Bent N, Tennant T, Swift T, Posnett J, Scuffham P, Chamberlain MA, et al. Team approach versus ad hoc health services for young people with physical disabilities: a retrospective cohort study. Lancet. 2002;360:1280-6.
18. Ladner TR, Mahdi J, Attia A, Froehler MT, Le TM, Lorinc AN, et al. A multispecialty pediatric neurovascular conference: a model for interdisciplinary management of complex disease. Pediatric Neurol. 2016;52:165-73.

19. Geerlings R, Aldenkamp AP, Gottmer-Welschen LM, et al. Long-term effects of a multidisciplinary transition intervention from paediatric to adult care in patients with epilepsy. Seizure. 2016;38:46-53.

20. Paganoni S, Nicholson K, Leigh F, et al. Developing multidisciplinary clinics for neuromuscular care and research. Muscle Nerve. 2017 Nov;56(5):848-58.

21. Busner J, Targum SD. The Clinical Global Impressions Scale: Applying a Research Tool in Clinical Practice. Psychiatry (Edgmont). 2007;4:28-37.

22. Zutt R, van Egmond ME, Elting JW, van Laar PJ, Brouwer OF, Sival DA, et al. A novel diagnostic approach to patients with myoclonus. Nat Rev. Neurol. 2015;12:687-97.

23. Egmond ME, Elting JWJ, Kuiper A, Zutt R, Heineman KR, Brouwer OF, et al. Myoclonus in childhood-onset neurogenetic disorders: The importance of early identification and treatment. Eur J Paediatr Neurol. 2015;19:726-9.

24. Liow NY, Gimeno H, Lumsden D, Marianczak J, Kaminska M, Tomlin S, et al. Gabapentin can significantly improve dystonia severity and quality of life in children. Eur J Paediatr Neurol. 2016;20:100-7.

\section{Submit your next manuscript to BioMed Central and we will help you at every step:}

- We accept pre-submission inquiries

- Our selector tool helps you to find the most relevant journal

- We provide round the clock customer support

- Convenient online submission

- Thorough peer review

- Inclusion in PubMed and all major indexing services

- Maximum visibility for your research

Submit your manuscript at www.biomedcentral.com/submit
Biomed Central 